\title{
Memoryless Predistortion of Nonlinear Amplifiers Based on Fourier Series Based Models
}

\author{
A. Pagès-Zamora, Miguel A. Lagunas, Tomás Jiménez \\ Dpt.Teoria Senyal i Comunicacions, ETSE Telecom., Universitat Politècnica de Catalunya (UPC) \\ Campus Nord UPC, Ed. D-5. c/ Gran Capità, s/n. 08034 Barcelona, Spain. \\ e-mail: alba@gps.tsc.upc.es
}

\begin{abstract}
*
In order to maximise the efficiency of the RF amplifier located in a transmitter, for instance in both analog and digital terrestrial TV links, it is forced to work near saturation introducing thus an undesirable nonlinear effect. A common solution includes a predistortion system before the modulation that compensates as much as possible the posterior nonlinear distortion, in such a way that the overall performance of the transmitter results in a iinear and efficient amplifier. Polynomial models usually implement the predistortion, but in this paper we propose an alternative model based on the Fourier-exponential series that shows better performance in the design stage without a significant increase of the complexity.
\end{abstract}

\section{Introduction}

The distortion introduced by a High Power Amplifier (HPA) located in the transmitter of a terrestrial link is usually equalised by the so-called feed-forward method, the negative feedback method or the predistortion method. The first one has a cost limitation since it needs two HPAs, which are quite expensive elements of the RF link. The so-called negative feedback method is another RF or intermediate frequency solution, which has an inherent instability problem. On the contrary, the predistortion method has no loop (avoiding thus any instability) and it results in a cheap solution since it can be implemented at the baseband level by a DSP. Nevertheless, in order to be able to apply the predistortion at the symbol level, several aspects should be taken into account. First of all, the fact that the HPA is located in the transmitter ensures the introduced nonlinear distortion is memoryless. This property, along with the bandpass behaviour of the HPA [1], allows a lowpass equivalent formulation where the HPA is completely characterised by the so-called AM/AM and $A M / P M$ curves which relates the input amplitude to the output amplitude and output phase, respectively. These curves are supposed to be independent of the frequency for narrow bandpass signals and they can be obtained by measuring the output of the HPA when it is

*This work was supported by PRONTIC/CICYT TIC-95-1022-C05-01 and CIRITr"Generalitat de Cataluny'a" GRQ93-3021 driven with a pure tone of the carrier frequency. The $\mathrm{AM} / \mathrm{AM}$ and $\mathrm{AM} / \mathrm{PM}$ relations are needed for the design stage, where the parameters of the predistortion are set. In general, a memoryless Volterra system is chosen to model the predistortion, being its coefficients fitted by an adaptive learning that is applied periodically before the data transmission due to the slow-time variation in the HPA characteristics.

In this paper, the authors propose an alternative system to model the predistortion that shows better performance in adaptive designs than the Volterra model does. Section 2 is thus devoted to present this model, which is based on a Fourier series development. In Section 3, the particular HPA predistortion problem is focused emerging the role and design of the memoryless nonlinear models. Finally, the simulation results are included in Section 4 where the performance of the Fourier versus the Volterra model in this particular topic is compared.

\section{The Fourier model}

The Fourier model arises from the Fourier series development of the input/output relation of the actual nonlinear system (NLS). If $\mathrm{g}[\mathrm{x}]$ denotes the relation of a given memoryless NLS, and $x$ is the input, the approximation of a $\mathrm{N}$-order Fourier is the following one.

$$
g[x] \approx \hat{g}[x]=\sum_{n=-N}^{N} c_{n} \cdot e^{j n \omega_{0} x}
$$

It is important to remark that, in order to avoid aliasing in the approximation provided by the Fourier model, the input signal $x$ should be bounded, i.e. $x \in[-$ $\left.\mathrm{X}_{\max }, \mathrm{X}_{\max }\right]$, being also the principal frequency upperbounded.

$$
\omega_{0}=\frac{2 \pi}{2 X_{0}} \leq \frac{2 \pi}{2 X_{\max }}
$$

Some previous works about the Fourier model have been already done, even with nonlinear problems with memory $[2,3]$. Without going into details, an important feature of this model is the fact that, once the order $\mathrm{N}$ and the principal frequency $\omega 0$ are chosen, the model is linear with the rest of coefficients, $\left\{c_{n}\right\}$. This property allows a MMSE criterion for designing the coefficients $\left\{c_{n}\right\}$ and, 
moreover, the use of classical adaptive methods to lead the model to this solution can be also applied [4].

In the HPA predistortion problem, the simplified model versions that consider an even or odd symmetry in the NLS input/output relation are specially interesting. Thus, the Fourier model allows a simplification when $\mathrm{g}[\mathrm{x}]$ has an odd (eq.3.a) or an even (eq.3.b) symmetry. The complexity is considerably reduced in comparison with the general Fourier model (eq.1) due to the real character of the coefficients and the functions.

$$
\begin{aligned}
& \text { If } g[x]=-g[-x] \Rightarrow \hat{g}(x)=\sum_{n=1}^{N} a_{n} \cdot \sin \left(n \omega_{0} x\right) \\
& \text { If } g[x]=g[-x] \Rightarrow \hat{g}(x)=\sum_{n=0}^{N} b_{n} \cdot \cos \left(n \omega_{0} x\right)
\end{aligned}
$$

Before dealing with the HPA predistortion problem, it is interesting to compare the Fourier model and the Volterra model in terms of complexity. Thus, an $\mathrm{N}$-order Volterra model needs of the order of $\mathrm{O}(\mathrm{N})$ multiplications to provide the successive powers of the input signal, whereas an $\mathrm{N}$-order Fourier model needs $\mathrm{O}(4 \mathrm{~N})$ real multiplications to compute the successive powers of the first order complex exponential, $\exp (j \omega \circ \mathrm{x})$. In fact, the memoryless Fourier model can be basically viewed as an $\mathrm{N}$-order Volterra model preceded by an exponential transformation (fig.1) and, in consequence, both models involve the same order of operations to generate the respective input data space (apart from the cost of computing the first complex exponential function).

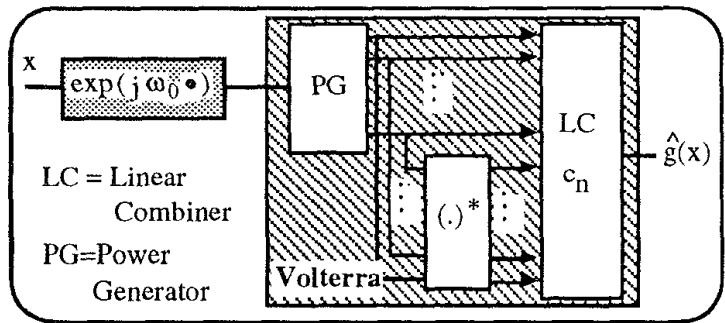

Figure 1. The memoryless Fourier model implementation

In case of dealing with the symmetric models, the Volterra model uses the half of the operations, whereas the Fourier model (eq.3.a,3.b) needs the same because the cosine/sine functions are obtained as the real/imaginary parts of the respective exponential functions. Concerning the number of coefficients that determine the computation load in the adaptive design, the Volterra model has $\mathrm{N}$ coefficients and the Fourier model has $(2 \mathrm{~N}+1)$ complex ones. Nevertheless, this number for the symmetric Volterra model is N/2, and for the Fourier Cosine or Sine models becomes $\mathrm{N}$ real coefficients.

\section{The HPA predistortion problem}

Let's consider a digital link with a transmitter as the one shown in figure (2). In a practical situation, the predistortion is located before the modulation, being usually designed by means of an adaptive method applied previously to the data transmission.

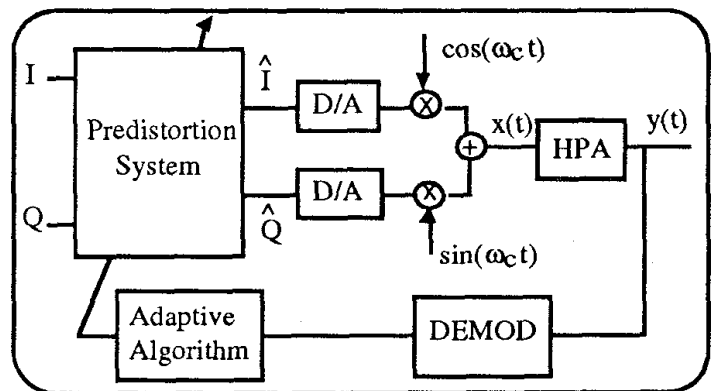

Figure 2. Adaptive learning of the predistortion system.

The input to the HPA is denoted by $x(t)$ and results in a narrow bandpass signal, centred round the frequency $\omega_{c}$ with an instantaneous amplitude and phase represented by, $R_{t}$ and $\theta_{t}$, respectively. Thus, the output of the HPA can be approximated by the following bandpass signal,

$y(t)=H P A[x(t)]=F\left[R_{t}\right] \cdot \cos \left(\omega_{c} t+\theta_{t}+\phi\left[R_{t}\right]\right)$

which involves the functions $F[R]$ and $\phi[R]$ that represent the so-called AM/AM and AM/PM relations of the HPA. In the simulations, these functions approximate the actual $\mathrm{AM} / \mathrm{AM}$ and AM/PM curves proposed in [5]. Whereas the amplitude distortion (fig.5.a) follows the relation,

$$
F[R]=\operatorname{sign}(R) * 0.62 *\left(1-\exp \left(-R^{2} / 0.25\right)\right)
$$

the phase predistortion $\phi[R]$ (fig.5.b) is implemented by an even polynomial with 9 coefficients.

The actual HPA output (eq.4) makes evident the fact that these curves completely characterise the HPA and, moreover, that they can be seen as a lowpass equivalent transformations. Thus, as it is shown in figure (3) for a discrete system, the predistortion design allows a lowpass formulation useful not only to find the design equations, but also for the simulations since we have not available real data.

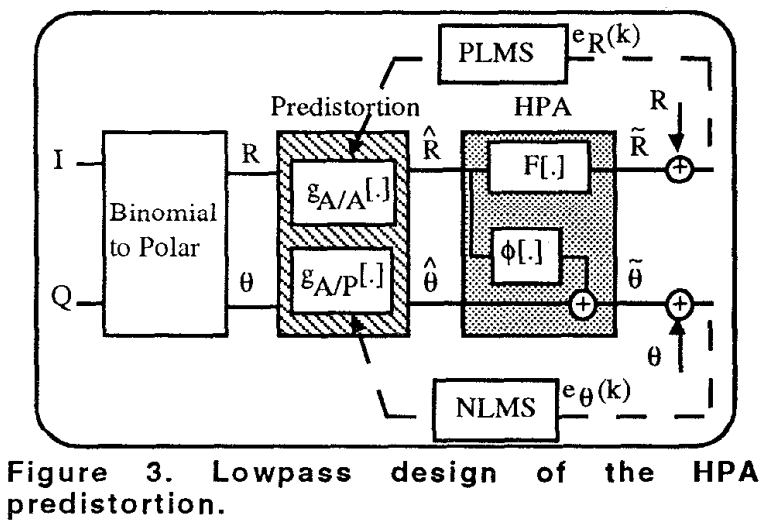


Concerning the amplitude predistortion denoted by gA/A[.], it can be designed in order to minimise the amplitude error $e_{R}(k)$, defined as the difference between the HPA AM/AM output and the desired magnitude $R_{k}$ (note that the HPA amplification is an scale factor, not included in the predistortion design).

$$
e_{R}(k)=R_{k}-F\left[\hat{R}_{k}\right]=R_{k}-F\left[g_{A / A}\left[R_{k}\right]\right] \approx 0
$$

Thus, ideally, the relation gA/A[R] should be the inverse transformation of $F[R]$. With respect to the phase predistortion denoted by the function $\mathrm{gA}_{\mathrm{P}}[\mathrm{l}$.], note that the minimisation of the phase error,

$$
e_{\theta}(k)=\theta_{k}-\hat{\theta}_{k}+\phi\left[\hat{R}_{k}\right] \approx 0 \Rightarrow g_{A / P}\left[\hat{R}_{k}\right] \approx-\phi\left[\hat{R}_{k}\right]_{(7)}
$$

conveys to a basic identification problem since the phase predistortion is applied after the amplitude predistortion.

It is important to remark that, although some noise will be present at the output of the actual HPA, the predistortion design equations derived from expressions (6) and (7) are useful since the main goal of the predistortion is to compensate nonlinearities, without taking care of the noise. In the simulations, an additive Gaussian noise at the output of the HPA curves will be considered with a high SNR (as it usually happens in terrestrial RF transmission) that allows a good performance of the proposed predistortion design.

\subsection{The Predistortion Models}

The odd symmetry of the AM/AM curve determines the model that implements the amplitude predistortion. In fact, the function gA/A[.] should also follow an odd relation and two possible models arise from the respective Volterra or Fourier series.

$$
\begin{aligned}
& g_{A / A}^{V}\left[R_{k}\right]=\sum_{n=1}^{N} a_{k}^{V}(n) \cdot R_{k}{ }^{(2 k-1)}=\left(a_{k}^{V}\right)^{\prime} \cdot u_{k}^{V} \\
& g_{A / A}^{F S}\left[R_{k}\right]=\sum_{n=1}^{N} a_{k}^{F S}(n) \cdot \sin \left(n \omega_{0} R_{k}\right)=\left(a_{k}^{F S}\right)^{\prime} \cdot u_{k}^{F S}
\end{aligned}
$$

The linear dependence of both models allows a vector notation in terms of the coefficient vector ak and the data vector $\mathbf{u}_{\mathrm{k}}$, which gathers the power functions for the Volterra model and the sine functions for the Fourier-Sine model. The coefficients in both models are time dependent since they are modified during the learning stage in order to minimise the power of the amplitude error. In [5], a kind of gradient adaptive algorithm is considered to update the value of the coefficients in the opposite direction of the instantaneous gradient. The resulting adaptive algorithm is called predistortion LMS (PLMS) algorithm and it involves the gradient of the AM/AM characteristic of the HPA with respect to the input value.

$$
\boldsymbol{a}(k+1)=\boldsymbol{a}(k)+\left.\mu e_{R}(k) \frac{\partial F(x)}{\partial x}\right|_{x=\hat{R}_{k}} u(k)
$$

In the simulations, the exact gradient of the proposed function F[.] has been used although in a real situation it can be also estimated in sections and stored in a table.

Similarly, the AM/PM curve follows an even relation and thus, the phase predistortion system should be also even. An even memoryless Volterra model (eq.10.a) and also a Fourier-Cosine model (eq.10.b) both of order $\mathrm{N}$ are proposed to implement the phase predistortion denoted by gA/P[.].

$$
\begin{aligned}
& g_{A / P}^{V}\left[\hat{R}_{k}\right]=\sum_{n=0}^{N-1} b_{k}^{V}(n) \cdot \hat{R}_{k}^{2 k}=\left(b_{k}^{V}\right)^{\prime} \cdot v_{k}^{V} \\
& g_{A / P}^{F C}\left[\hat{R}_{k}\right]=\sum_{n=0}^{N-1} b_{k}^{F C}(n) \cdot \cos \left(n \omega_{0} \hat{R}_{k}\right)=b_{k}^{F C^{\prime}} \cdot v_{k}^{F C}
\end{aligned}
$$

The update equations for the coefficients $b_{k}$ involved in the phase predistortion models can be performed by classical adaptive methods (the NLMS algorithm is proposed), since the design problem consists in a basic identification problem with a model that is linear in terms of its coefficients.

$$
\boldsymbol{b}(k+1)=\boldsymbol{b}(k)+\frac{\mu}{p(k)} e_{\theta}(k) \boldsymbol{v}(k)
$$

The term $\mathrm{p}(\mathrm{k})$ denotes the estimated input power approximated by a lowpass filtering of the input data vector $\mathrm{vk}$ with a memory factor named $\beta$. It is interesting to note that, since the output of the amplitude predistortion drives the phase predistortion, the learning of the coefficients of the gA/P [.] model will be conditioned to the learning of the amplitude predistortion system.

\section{Simulation Results}

In this section, the results obtained in the simulation of the adaptive learning of the predistortion ( $f i g .3$ ) are presented. The input signal is a 64-QAM modulation generated from two 8-PAM signals, for the in-phase $\mathrm{I}_{k}$ and the in-quadrature $Q_{k}$ components. The resulting magnitude should be less than 0.62 , which is the range capable of being compensated since the output of the normalised F[.] function of the BIT transistor is bounded to this range (eq.5). A Gaussian noise $(\mathrm{SNR}=60 \mathrm{~dB})$ is also added to the output of the HPA distortion.

Concerning the amplitude distortion, gA/A[.], two different models are considered: an odd Volterra (oV) model (eq.8.a) and a Fourier-Sine (FS) model (eq.8.b), both with $\mathrm{N}=5$ coefficients. The principal frequency for the Fourier model is chosen equal to $\omega 0=\pi /(2 * 0.62)$ since the input value $R$ is bounded to 0.62 . The coefficients are updated by the PLMS algorithm (eq.9), where the step size parameters are normalised to the power estimate of the respective data vector $\mathbf{u}\left(\mu_{\mathrm{oV}}(\mathrm{k})=0.003 /\left(\mathrm{p}_{\mathrm{oV}}(\mathrm{k})\right)\right.$ with $\operatorname{pov}(-1) \approx 0, \mu \mathrm{FS}(\mathrm{k})=2 / \mathrm{N})$. At this point it is worth valued to remark that the diversity managed by the Fourier model, i.e. the sine functions of successive harmonics, has not so scattered power values as the Volterra model. 
This feature, together with other aspects concerning the correlation between the components of the vector $\mathbf{u}_{\mathrm{k}}[3]$, usually provides the Fourier model with a better performance than the Volterra model in adaptive solutions. A clear example is shown in figure (4.a) that represents the squared amplitude error achieved by both models averaged over 25 independent realisations (the better result is achieved by the FS model). The step-sizes have been fitted after various tests to achieve a similar convergence rate for both models. Additionally, the amplitude predistortion system implemented by the Fourier model and Volterra model after the learning stage are included in figure (5.c).
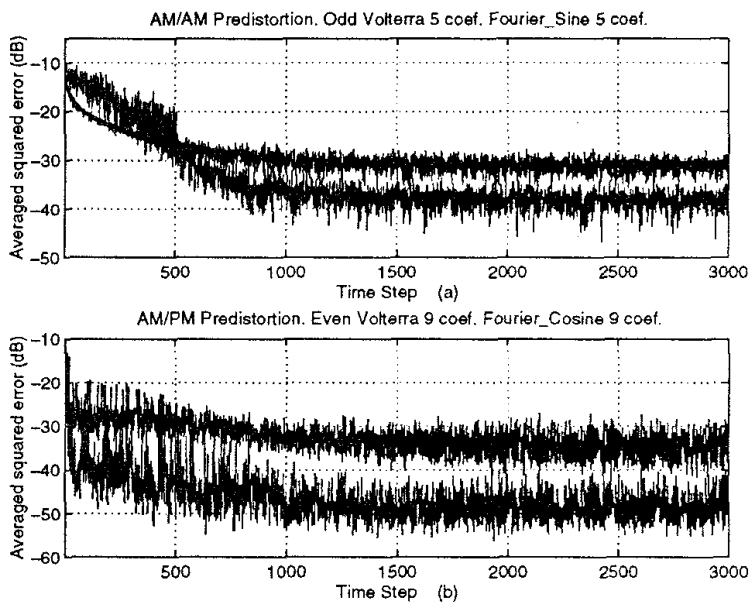

Figure 4. Squared envelope error (a) and squared phase error (b) averaged over 25 realisations.

Similarly, the averaged squared phase error achieved by the AM/PM predistortion is included in figure (4.b) (the Fourier model also shows the better results). For this nonlinear system, an even Volterra $(\mathrm{eV})$ model and a Fourier-Cosine (FC) model with 9 coefficients are used. The respective coefficients are updated by the NLMS algorithm (eq.11) with the following step-sizes ( $\mu \mathrm{Ve}=0.05$ pVe $(-1) \approx 0, \mu \mathrm{FC}=0.3 \mathrm{pFC}(-1)=1)$. In this case, the principal frequency of the Fourier model is chosen equal to $\omega_{0}=\pi /(2)$ because the input to our model, denoted by ${ }^{\wedge} \mathrm{R}_{\mathrm{k}}$ is bounded to one. At the beginning of the learning, this assumption does not hold and the phase predistortion learning could be in troubles. Thus, in the simulations, the output of the amplitude predistortion system is forced to be less than one in order to avoid this problem. From the error performance, it can be seen how the phase predistortion is conditioned to the convergence of the amplitude predistortion as it was expected. Finally, the AM/PM predistortion implemented by both models after the learning stage are also shown in figure (5.d), where the superior performance of the Fourier model becomes evident.
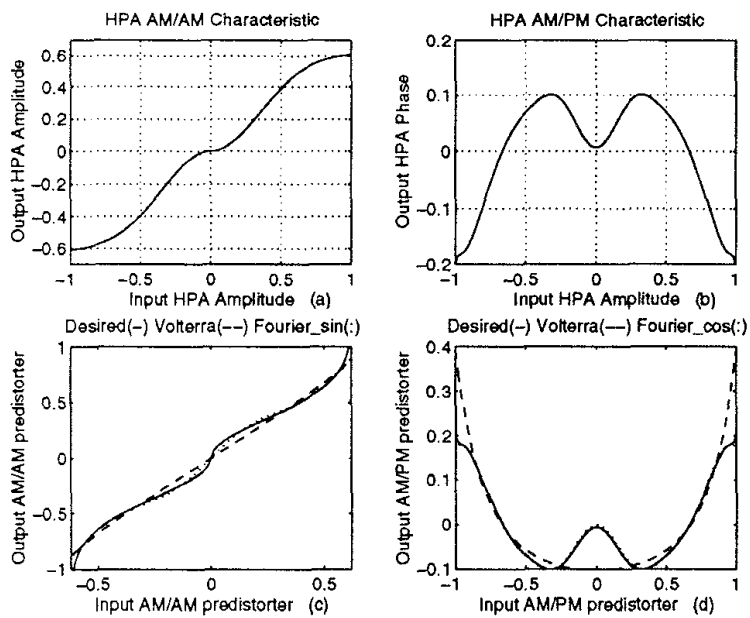

Figure 5. (a) HPA AM/AM relation. (b) HPA $A M / P M$ relation. (c) Ideal amplitude predistortion (solid) and final amplitude predistortion of the Fourier-Sine model (dotted) and odd Volterra model (dashed). (d) Ideal phase predistortion (solid) and final phase predistortion of the Fourier-Cosine model (dotted) and odd-Volterra model (dashed).

\section{Remarks}

In this paper the HPA predistortion implemented by a Fourier model is compared with the performance achieved by the classical solution of using polynomial models. Although the Fourier model requires more computational load than the Volterra model, the existing fast DSP processors as well as the considerably superior performance achieved in this particular problem by the Fourier model seem to justify the use of this last one.

\section{References}

[1] Benedetto S., Biglieri E., Castellani V., "Digital Transmission Theory", Prentice-Hall, Inc., Englewood Cliffs, New Jersey, 1987.

[2] Pagès-Zamora A.,Lagunas M.A., Nájar M., Pérez-Neira A., "The K-filter: A new architecture to model and design non-linear systems from Kolmogorov's theorem", EURASIP Signal Processing, Vol.44, No.3, pp.249267, July 1995.

[3] Pagès-Zamora A., Lagunas M.A., "Joint Probability Density Function Estimation by Spectral Estimate Methods", Proc. IEEE-ICASSP'96, Atlanta, Georgia, USA, May 1996.

[4] Mathews V.J., "Adaptive Polynomial Filters", IEEE Signal Processing Magazine, V.8, No3, pp.10-26, July 1991.

[5] Stonick I.T. et al., "Memoryless Polynomial Adaptive Predistortion", Proc. IEEE-ICASSP'95, pp.981-984, Detroit, Michigan, USA, May 1995. 